\title{
AN UNCONVENTIONAL SUPERGRAVITY
}

\author{
PAVEL GROZMAN, DIMITRY LEITES
}

\begin{abstract}
We introduce and completely describe the analogues of the Riemann curvature tensor for the curved supergrassmannian of the passing through the origin (0|2)-dimensional subsupermanifolds in the (0|4)-dimensional supermanifold with the preserved volume form. The underlying manifold of this supergrassmannian is the conventional Penrose's complexified and compactified version of the Minkowski space, i.e., the Grassmannian of 2dimensional subspaces in the 4-dimensional space.

The result provides with yet another counterexample to Coleman-Mandula's theorem.
\end{abstract}

This paper should appear in: S. Duplij and J. Wess (eds.) Noncommutative structures in mathematics and physics, Proc. NATO Advanced Reserch Workshop, Kiev, 2000. Kluwer, $13-30$.

1. New supertwistors. Penrose suggested an unusual description of our space-time, namely, to compactify the Minkowski space-time model of the Universe (nontrivially: with a light cone at the infinity) and complexify this compactification. The final result is $G r_{2}^{4}$, the Grassmanian of 2-dimensional subspaces in the 4-dimensional (complex) space (of socalled twistors). There are many papers and several monographs on advantages of this interpretation of the space-time in various problems of mathematical physics; we refer the reader to Manin's book [5], where an original Witten's idea to incorporate supervarieties and consider infinitesimal neighborhoods for interpretation of the "usual", i.e., non-super, YangMills equations is thouroghly investigated together with several ways to superize Minkowski space. Ours is one more, distinct, way.

Observe that the supermanifold of (0|2)-dimensional subsuperspaces in the (0|4)-dimensional superspace is identical with $G r_{2}^{4}$, only the tautological bundle is different: the fiber is purely odd. In this work we consider not subsuperspaces but subsupermanifolds.

We considered the structure functions - analogs of the Riemann tensor - for the curved supergrassmannian $\mathcal{C} G r_{0 \mid 2}^{0 \mid 4}$ of (0|2)-dimensional subsupermanifolds in the (0|4)-dimensional supermanifold. Recall that the "usual" grassmannian consists of linear subspaces of the linear space passing through the origin whereas the curved one consists of submanifolds, in other words, nonlinear embeddings are allowed and the submanifolds do not have to pass through a fixed point. Obviously, the curved Grassmannian is infinite dimensional, but the curved supergrassmannian $\mathcal{C} G r_{0, k}^{0, n}$ is of finite superdimension: it is a quotient of the supergroup of superdiffeomorphisms of the linear supermanifold $\mathcal{C}^{0, n}$ (the Lie superalgebra of this Lie supergroup is $\left.\operatorname{vect}(0 \mid n)=\mathfrak{d} \mathfrak{e r} \mathbb{C}\left[\theta_{1}, \ldots, \theta_{n}\right]\right)$. For the list of classical superspaces including curved supergrassmannians see [4].) The underlying manifold of $\mathcal{C} G r_{0 \mid 2}^{0 \mid 4}$ is the conventional $G r_{2}^{4}$ but $\mathcal{C} G r_{0,2}^{0,4}$ has also odd coordinates.

On $\mathcal{C} G r_{0 \mid 2}^{0 \mid 4}$, we have expanded the curvature supertensor in components with respect to the (complexification of the) Lorentz group and saw that it does not contain the components used for the ordinary Einstein equations (EE), namely, there is no Ricci curvature Ric and no scalar curvature Scalar (in what follows $R(22)$ and $R(00)$, respectively).

We gratefully acknowledge partial financial support of The Swedish Institute and an NFR grant, respectively. 
So we decided to amend the initial model and consider the supergrassmanian $\mathcal{C} G r_{0 \mid 2}^{0 \mid 4}(0)$ of subsupermanifolds through the origin. It turns out that this does not help, either: no Ric and Scalar.

We decided not to give up, and took for the model of Minkowski superspace the supergrassmannian $\mathcal{S} C G r_{0 \mid 2}^{0 \mid 4}(0)$ of subsupermanifolds through the origin with the volume element of the ambient and the subsupermanifolds preserved. On $\mathcal{S} C G r_{0 \mid 2}^{0 \mid 4}(0)$, the expansion of the curvature supertensor does contain $R(22)$ and $R(00)$ ! There are no analogs of conformal (off shell) structure functions.

Our model and its supergroup of motion — an analogue of the Poincaré group — do not contradict the restrictions of the famous no-go theorems by Haag-Lopuszanski-Sohnius and Coleman-Mandula (for further discussions see [1]) and provides us with a new, missed so far, version of the Poincaré supergroup. The analogues of Einstein equations we suggest are a totally new version of SUGRA. Equating to zero other conformally non-invariant components we get extra conditions; we do not know how to interprete them.

We do not see any reason for discarding this and similar models. In particular, we suggest to analyze the structurre functions (definition below) on $\mathcal{C} G r_{0 \mid 2}^{0 \mid 4}$ and $\mathcal{C} G r_{0 \mid 2}^{0 \mid 4}(0)$ which we have abandoned above.

The conventional reading of Coleman-Mandula's theorem (cf. [6]) assumes that the complexified Lorentz Lie algebra $\mathfrak{L}=\mathfrak{s l}(2)_{L} \oplus \mathfrak{s l}(2)_{R}$ commutes with the Lie algebra of internal symmetries $\mathfrak{i}$ (for us $\mathfrak{i}$ is equal to $\mathfrak{s l}(2)_{L} \otimes \mathbb{C} \xi_{1} \xi_{2}$, see sec. 4).

In our case $\mathfrak{L}$ acts on $\mathfrak{i}$ and forms a semidirect sum with it; the bracket on $\mathfrak{i}$ is identically zero. This possibility does not contradict assumptions of Coleman-Mandula's theorem but was not considered.

The odd parameters have a correct statistics with respect to the Lorentz Lie algebra.

We represent Einstein's equations as conditions on conformally noninvariant components of the analog of the Riemann tensor, and represent the Riemann tensor as a section of the bundle on the (locally) Minkowski space whose fiber is certain Lie algebra cohomology. This is a more user-friendly description of the Riemannian tensor than the classical treatment of obstructions to nonflatness in differential geometry. We have in mind Spencer homology, cf. [7], where the case of any $G$-structure, not only $G=O(n)$ is considered. Superization of the definitions from [7] is the routine straightforward application of the Sign Rule.

Remark. It is interesting to test the whole list of curved supergrassmannians with the simple Lie supergroup of motion (see Tables in [4]) and similarly to the above sacrify the simplicity of the supergroup of motion in order to get EE. Grozman's package SuperLie (see [2]) is a useful tool in this research problem: without a computer (and a good code) this task is hardly feasible.

2. Structure functions: recapitulation $([\overline{7}])$. Let $F(M)$ be the frame bundle over a manifold $M$, i.e., the principal $G L(n)$-bundle. Let $G \subset G L(n)$ be a Lie group. A $G$-structure on $M$ is a reduction of the principal $G L(n)$-bundle to the principal $G$-bundle.

The simplest $G$-structure is the flat $G$-structure defined as follows. Let $V$ be $\mathbb{R}^{n}$ (or $\mathbb{C}^{n}$ ) with a fixed frame. The flat structure is the bundle over $V$ whose fiber over $v \in V$ consists of all frames obtained from the fixed one under the $G$-action, $V$ being identified with $T_{v} V$ by means of the parallel translation by $v$.

Examples of flat structures. The classical spaces, e.g., compact Hermitian symmetric spaces, provide us with examples of manifolds with nontrivial topology but flat $G$-structure.

In [7] the obstructions to identification of the $k$ th infinitesimal neighbourhood of a point $m$ on a manifold $M$ with $G$-structure with the $k$ th infinitesimal neighbourhood of a point of the flat manifold $V$ with the above described flat $G$-structure are called structure functions 
of order $k$. In [7] it is shown further that the tensors that constitute these obstructions are well-defined provided the structure functions of all orders $<k$ vanish. (In supergravity the conditions that structure functions of lesser orders vanish are called Wess-Zumino constraints.)

The classical description of the structure functions uses the notion of the Spencer cochain complex. Let us recall it. Let $S^{i}$ denote the operator of the $i$-th symmetric power. Set $\mathfrak{g}_{-1}=T_{m} M$, let $\mathfrak{g}_{0}$ be the Lie algebra of $G$; for $i>0$ set:

$$
\begin{gathered}
\mathfrak{g}_{i}=\left\{X \in \operatorname{Hom}\left(\mathfrak{g}_{-1}, \mathfrak{g}_{i-1}\right) \mid X\left(v_{0}\right)\left(v_{1}, \ldots, v_{i}\right)=X\left(v_{1}\right)\left(v_{0}, \ldots, v_{i}\right)\right. \\
\text { for any } \left.v_{0}, v_{1}, \ldots, v_{i} \in \mathfrak{g}_{-1}\right\} .
\end{gathered}
$$

Finally, set $\left(\mathfrak{g}_{-1}, \mathfrak{g}_{0}\right)_{*}=\underset{i \geq-1}{\bigoplus} \mathfrak{g}_{i}$. This is the Lie algebra of all transformations that preserve on $\mathfrak{g}_{-1}$ the same structure which is preserved by the linear transformations from $\mathfrak{g}_{0}$.

Suppose that the $\mathfrak{g}_{0}$-module $\mathfrak{g}_{-1}$ is faithful, i.e., each nonzero element from $\mathfrak{g}_{0}$ acts nontrivially. Then, clearly,

$$
\left(\mathfrak{g}_{-1}, \mathfrak{g}_{0}\right)_{*} \subset \mathfrak{v e c t}(n)=\mathfrak{d e r} \mathbb{R}\left[x_{1}, \ldots, x_{n}\right],
$$

where $n=\operatorname{dim} \mathfrak{g}_{-1}$, with

$$
\mathfrak{g}_{i}=\left\{X \in \mathfrak{v e c t}(n)_{i}:[X, D] \in \mathfrak{g}_{i-1} \text { for any } D \in \mathfrak{g}_{-1}\right\}
$$

for $i \geq 1$. It is easy to check that $\left(\mathfrak{g}_{-1}, \mathfrak{g}_{0}\right)_{*}$ is a Lie subalgebra of $\mathfrak{v e c t}(n)$.

The Lie algebra $\left(\mathfrak{g}_{-1}, \mathfrak{g}_{0}\right)_{*}$ will be called the Cartan's prolong (the result of Cartan's prolongation) of the pair $\left(\mathfrak{g}_{-1}, \mathfrak{g}_{0}\right)$.

Let $E^{i}$ be the operator of the $i$-th exterior power; set (prime denotes dualization)

$$
C_{\left(\mathfrak{g}_{-1}, \mathfrak{g}_{0}\right)}^{k,}=\mathfrak{g}_{k-s} \otimes E^{s}\left(\mathfrak{g}_{-1}^{\prime}\right)
$$

Define the differential $\partial_{s}: C_{\left(\mathfrak{g}_{-1}, \mathfrak{g}_{0}\right)}^{k, s} \longrightarrow C_{\left(\mathfrak{g}_{-1}, \mathfrak{g}_{0}\right)}^{k, s+1}$ by setting for any $v_{1}, \ldots, v_{s+1} \in V$ (as usual, the slot with the hatted variable is to be ignored):

$$
\left(\partial_{s} f\right)\left(v_{1}, \ldots, v_{s+1}\right)=\sum(-1)^{i}\left[f\left(v_{1}, \ldots, \hat{v}_{s+1-i}, \ldots, v_{s+1}\right), v_{s+1-i}\right] .
$$

As expected, $\partial_{s} \partial_{s+1}=0$, and the homology $H_{\left(\mathfrak{g}_{-1}, \mathfrak{g}_{0}\right)}^{k, s}$ of the bicomplex $\underset{k, s}{\oplus} C_{\left(\mathfrak{g}_{-1}, \mathfrak{g}_{0}\right)}^{k, s}$ is called the $(k, s)$-th Spencer cohomology of $\left(\mathfrak{g}_{-1}, \mathfrak{g}_{0}\right)_{*}$. (Observe that we use a grading of the Spencer complex different form that in [7]. Ours is a more natural one.)

Proposition ([7]) The structure functions of order $k$ constitute the space of the $(k, 2)-t h$ Spencer cohomology of the $\left(\mathfrak{g}_{-1}, \mathfrak{g}_{0}\right)_{*}$.

3. Spencer cohomology in terms of Lie algebra cohomology. We observe that

$$
\underset{k}{\oplus} H_{\left(\mathfrak{g}_{-1}, \mathfrak{g}_{0}\right)}^{k, 2}=H^{2}\left(\mathfrak{g}_{-1} ;\left(\mathfrak{g}_{-1}, \mathfrak{g}_{0}\right)_{*}\right) .
$$

The advantage of this reformulation: the Lie algebra cohomology (the right hand side of (3)) is easier to compute (e.g., by means of the package SupeLie when the general theory fails, or with the help of various theorem). At the same time the fine grading of Spencer homology is not lost: the $\mathbb{Z}$-grading of $\left(\mathfrak{g}_{-1}, \mathfrak{g}_{0}\right)_{*}$ which induces the grading $(3)$ of $H^{2}\left(\mathfrak{g}_{-1} ;\left(\mathfrak{g}_{-1}, \mathfrak{g}_{0}\right)_{*}\right)$ coincides (up to a shift) with the oder of the structure functions.

Analogs of Weyl and Riemann tensors. Suppose $\mathfrak{g}_{0}$ contains a center (like in the case when a metric is preserved up to a conformal factor). Then the elements of $H^{2}\left(\mathfrak{g}_{-1} ;\left(\mathfrak{g}_{-1}, \mathfrak{g}_{0}\right)_{*}\right)$ are analogs of the Weyl tensor.

Let $\hat{\mathfrak{g}}_{0}$ be the semisimple part of $\mathfrak{g}_{0}$ and let $\hat{\mathfrak{g}}_{*}$ be a shorthand for $\left(\mathfrak{g}_{-1}, \hat{\mathfrak{g}}_{0}\right)_{*}$. The elements of $H^{2}\left(\mathfrak{g}_{-1} ; \hat{\mathfrak{g}}_{*}\right)$ are analogs of the Riemann tensor. 
The relation between $\hat{H}=H^{2}\left(\mathfrak{g}_{-1} ; \hat{\mathfrak{g}}_{*}\right)$ and $H=H^{2}\left(\mathfrak{g}_{-1} ;\left(\mathfrak{g}_{-1}, \mathfrak{g}_{0}\right)_{*}\right)$ is more intricate for the general $\hat{\mathfrak{g}}_{0}$ than in the Riemannian case $\left(\hat{\mathfrak{g}}_{0}=\mathfrak{o}(n)\right)$ when $\hat{H}$ strictly contains $H$. In general, these spaces have common components (conformally invariant, "on shell" ones) and have other components, analogs of "off shell" components, cf. [3].

In the Riemann case, there are two "off shell" components: with the highest weights $(2,2)$ (the traceless Ricci tensor) and $(0,0)$ (the scalar curvature). Here the highest weights are given with respect to the complexification $\mathfrak{L}=\mathfrak{s l}(2)_{L} \oplus \mathfrak{s l}(2)_{R}$ of the $\mathfrak{o}(1,3)$. The Einstein equaton (in vacum) is a vanishing condition of these components. Remarkably, there are no structure functions of lesser order. If they had existed, we would have to impose analogs of Wess-Zumino constraints to be able to define the usual Riemann curvature tensor.

4. The description of $\left(\mathfrak{g}_{-1}, \mathfrak{g}_{0}\right)_{*}$ for the curved supergrassmannians. For the general curved supergrassmannian of $(0, k)$-dimensional subsupermanifolds $\mathcal{S}$ in the $(0, n)$ dimensional supermanifold $\mathcal{T}$ let $\xi_{1}, \ldots, \xi_{k}$ be the coordinates of $\mathcal{S}$ and $\theta_{1}, \ldots, \theta_{n-k}$ the remaining coordinates of $\mathcal{T}$. Then setting $\operatorname{deg} x i_{i}=0$ for all $i$ and $\operatorname{deg} \theta_{j}=1$ for all $j$ we get a $\mathbb{Z}$-grading of $\mathfrak{v e c t}(0 \mid n)$ of the form

$$
\mathfrak{g}_{0}=(\mathfrak{g} l(V) \otimes \mathbb{C}[\xi]) \boxplus \mathfrak{v e c t}(\xi) ; \quad \mathfrak{g}_{-1}=V \otimes \mathbb{C}[\xi] ;
$$

where $V=\operatorname{Span}\left(\frac{\partial}{\partial \theta_{1}}, \ldots, \frac{\partial}{\partial \theta_{n-k}}\right)$ is the identity $\mathfrak{g} l(V)$-module, and $\boxplus$ is the sign of a semidirect sum of algebras: $\mathfrak{a} \boxplus \mathfrak{b}$ with the ideal $\mathfrak{a}$.

For $n=4$ we computed $H^{2}\left(\mathfrak{g}_{-1} ;\left(\mathfrak{g}_{-1}, \mathfrak{g}_{0}\right)_{*}\right)$ in the following cases:

(a) the general curved supergrassmannians;

(b) the supergrassmannians of subspaces through 0, i.e., we removed from vect all partial derivatives (since this is not an invariant formulation, it is better to say: we only considered the vector fields that vanish at the origin);

(c) in case (b) we only considered volume-preserving transformations, i.e., we diminished $\mathfrak{g}_{0}$ as well:

$$
\mathfrak{g}_{0}=(\mathfrak{s l}(V) \otimes \mathbb{C}[\xi]) \boxplus \mathfrak{s l}(\operatorname{Span}(\xi)) ; \quad \mathfrak{g}_{-1}=V \otimes \mathbb{C} \xi
$$

In particular, since $\mathfrak{g}_{-1}$ is isomorphic to the tangent space at a point of the curved supergrassmannian, we see that its even part in cases (a) - (c) is the same $G r_{2}^{4}$ while the tangent space to the whole supermanifold at the "origin" is $\operatorname{Span}\left(\xi_{i} \frac{\partial}{\partial \theta_{j}}: 1 \leq i, j \leq 2\right)$. So the number of odd coordinates of our model varies from 4 in case (a) to 2 in cases (b) and (c).

Table. In the first line there are indicated the degrees, i.e., orders, of all nonzero structure functions and the rest of the table lists their the weights (with respect to $\mathfrak{L}$ ) (superscript denotes the multiplicity of the weight the subscript the degree of the corresponding structure function). The $\mathfrak{g}_{0}$-action is nontrivial and glues distinct irreducible $\left(\mathfrak{g}_{0}\right)_{\overline{0}}$-modules. (We did not show the action though we have computed it.)

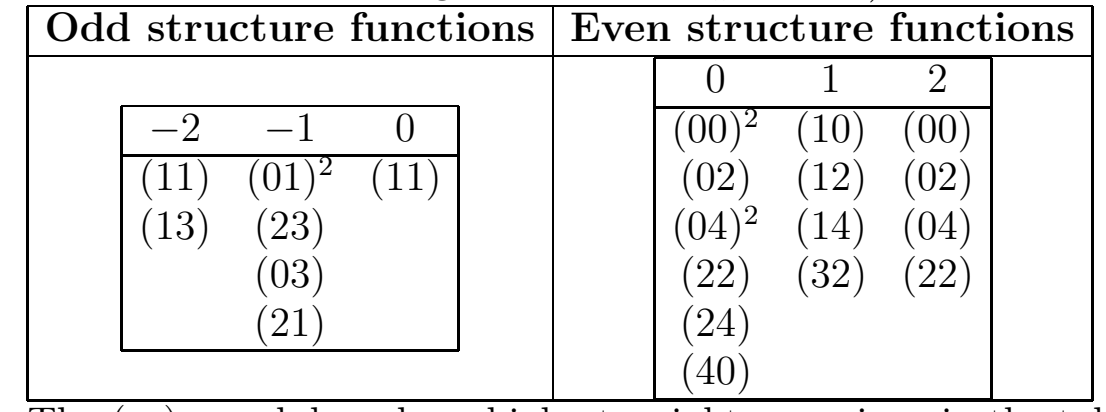

The $\left(\mathfrak{g}_{0}\right) \overline{0^{-}}$-modules whose highest weights are given in the table are glued into $\mathfrak{g}_{0}$-modules as follows (an arrow indicates a submodule). The even tensors:
$\searrow$
$(12)$
$(02)_{2}$
$(04)_{0}$
$\longrightarrow \quad(04)_{2}$;
$(14)_{1} \quad \nearrow$; 


$$
\begin{array}{cl}
(22)_{0} \longrightarrow(14)_{1} \longrightarrow(22)_{2} ; & (22)_{0} \longrightarrow(32)_{1} \longrightarrow(22)_{2} ; \\
(24)_{0} \longrightarrow(32)_{1} ;(12)_{1} \longrightarrow(04)_{2} ; \quad(40)_{0} \longrightarrow(32)_{1} ;(12)_{1} \longrightarrow(22)_{2} .
\end{array}
$$

The odd tensors:

$$
\begin{aligned}
& (11)_{-2} \longrightarrow(23)_{-1} ; \quad(01)_{-1}^{2} \longrightarrow(11)_{0} \\
& (13)_{-2} \longrightarrow(23)_{-1}
\end{aligned}
$$

5. The Einstein equations. The conventional EE in vacum are the conditions on the two tensors of degree 2 and weight (00) and (22), namely,

$$
R(22)=0 \quad \text { and } \quad R(00)=\lambda g,
$$

where $\lambda \in \mathbb{C}$ is interepreted in terms of the cosmological constant and $g$ is the metric preserved.

For an analog of the Einstein equations on the curved supergrassmannian we may take the same vanishing conditions of the 2-nd order structure functions of weights (00) and (22) with respect to $\mathfrak{L}$. However, unlike the Einstein's case, we have to vanish the constraints, the structure functions of lesser orders, both even and odd. The meaning of these analogs of Wess-Zumino constraints is unclear to us.

\section{REFERENCES}

[1] Deligne P. et al (eds.) Quantum fields and strings: a course for mathematicians. Vol. 1, 2. Material from the Special Year on Quantum Field Theory held at the Institute for Advanced Study, Princeton, NJ, 1996-1997. AMS, Providence, RI; Institute for Advanced Study (IAS), Princeton, NJ, 1999. Vol. 1: xxii+723 pp.; Vol. 2: pp. i-xxiv and 727-1501

[2] Grozman P., Leites D., Mathematica-aided study of Lie algebras and their cohomology. From supergravity to ballbearings and magnetic hydrodynamics In: Keränen V. (ed.) The second International Mathematica symposium, Rovaniemi, 1997, 185-192

[3] Grozman P., Leites D., Supergravities and N-extended Minkowski superspaces for any N. In: Wess J., Ivanov E. (eds.) Supersymmetries and quantum symmetries. Proc. International Conference in memory of V. Ogievetsky, June 1997, Lecture Notes in Physics 524, Springer, 1999, 58-67

[4] Leites D., Serganova V., Vinel G., Classical superspaces and related structures. In: Bartocci C. et al. (eds) Differential Geometric Methods in Theoretical Physics. Proc. DGM-XIX, 1990, Springer, LN Phys. 375, 1991, 286-297

[5] Manin, Yu. Gauge field theory and complex geometry, Springer-Verlag, Berlin, 1997.

[6] Salam A., Sezgin E., Supergravities in diverse dimensions, v.v. 1, 2, World Scientific, 1989

[7] Sternberg S., Lectures on differential geometry, Chelsey, 2nd edition, 1985

Department of Mathematics, University of Stockholm, Kräftriket hus 6, SE-106 91, Stockholm, Sweden 